ÉGYPTE monde arabe

\section{Égypte/Monde arabe}

$20 \mid 1994$

L'Égypte en débats

\title{
Péripéties et vicissitudes de la libéralisation du marché du travail en Égypte
}

\section{Françoise Clément}

\section{(2) OpenEdition}

\section{Journals}

Édition électronique

URL : https://journals.openedition.org/ema/522

DOI : $10.4000 /$ ema.522

ISSN : 2090-7273

\section{Éditeur}

CEDEJ - Centre d'études et de documentation économiques juridiques et sociales

\section{Édition imprimée}

Date de publication : 31 décembre 1994

Pagination : 143-153

ISSN : 1110-5097

\section{Référence électronique}

Françoise Clément, «Péripéties et vicissitudes de la libéralisation du marché du travail en Égypte », Égypte/Monde arabe [En ligne], 20 | 1994, mis en ligne le 08 juillet 2008, consulté le 07 juillet 2022. URL : http://journals.openedition.org/ema/522 ; DOI : https://doi.org/10.4000/ema.522

Ce document a été généré automatiquement le 7 juillet 2022.

Tous droits réservés 


\title{
Péripéties et vicissitudes de la libéralisation du marché du travail en Égypte
}

\author{
Françoise Clément
}

1 Le processus de « désétatisation » de l'activité économique et de la vie politique engagé depuis la fin des années 60 s'est traduit jusqu'à présent par des modifications mineures de la législation du travail de 1962, laissant intacts les fondements de la charte sociale instituée par la constitution provisoire de 1961. De fait, l'extraordinaire accroissement de la mobilité du travail induite par le boom pétrolier rendait inutile et inopérante toute modification du cadre législatif. Avec le programme d'ajustement structurel en cours, la refonte radicale de la législation du travail est à nouveau à l'ordre du jour. La baisse de la demande de travail en Égypte et à l'Étranger pousse les salariés à conserver leur emploi au moment où la restructuration du secteur public prévoit d'importantes réductions d'effectifs. De plus, la disparition des possibilités de pluriactivité fait du salaire l'unique source de revenus de la grande masse des salariés situés au bas de la hiérarchie professionnelle.

2 Or, le relèvement du cours des devises, des prix intérieurs et des taux d'intérêt a mis à nu l'endettement massif des entreprises publiques et privées, hérité de la vague d'investissement débridé qui a accompagné le boom pétrolier. Ne pouvant compter ni sur une annulation des dettes intérieures qui entraînerait une grave baisse de la rémunération des dépôts et une nouvelle fuite des capitaux, ni sur la baisse des prix de leurs intrants, ni sur une augmentation rapide de leurs ventes, les chefs d'établissement ne peuvent assainir leurs comptes qu'en réduisant les coûts salariaux et les gaspillages dus à une organisation du travail conçue pour un système de prix relatifs différents.

3 Le projet de réforme et d'unification de la législation du travail, impulsé par le Bureau international du travail (BIT) sur recommandation de la Banque mondiale, vise à réaliser ces deux objectifs. Suivant l'adoption, en 1991, des lois 203 et 230 de réorganisation des secteurs public et privé mixte, il s'inscrit dans un vaste ensemble de 
refonte législative des relations économiques et de travail, qui comprend également la réforme des lois régissant l'activité syndicale, les assurances sociales et les retraites. Dirigée par deux experts, un juriste du BIT et un universitaire égyptien, l'élaboration du nouveau code a mobilisé, outre des juristes spécialisés, les forces politiques et institutionnelles que le BIT et le gouvernement égyptien souhaitent associer aux nouvelles formes de gestion des relations de travail, à savoir l'Union des industries, les syndicats de branches et leur fédération ainsi que le Ministère du Travail, représentant respectivement les employeurs; les employés et l'État.

4 Les commissions syndicales d'entreprise et les partis politiques d'opposition n'ont été associés à l'élaboration du projet que de façon indirecte, à travers des réunions "d'écoute " auxquelles des délégués syndicaux appartenant aux courants politiques opposés au projet avaient été conviés. Le texte même du projet, officiellement tenu secret jusqu'au moment de sa présentation devant le Parlement, a fait l'objet, à différentes étapes de son élaboration, de «fuites» qui ont permis à la presse d'opposition nassérienne-marxiste et nassérienne-islamiste d'en publier des extraits et de se faire le relais des courants syndicaux opposés au nouveau code. C'est dans le contexte de l'élaboration du projet de code du travail, du secret qui l'entoure et des inquiétudes qu'il suscite dans les milieux ouvriers qu'il faut resituer le premier grand conflit du travail depuis la mise en œuvre de l'ajustement structurel. Déclenché à la fin du mois de septembre dernier dans l'entreprise "Misr pour la filature fine ", située à Kafr al-Dawwâr, il a été provoqué par une tentative d'application, par la direction de l'entreprise, de dispositions de la loi 203 de 1991 relatives à l'attribution des primes et aux interruptions de contrats à durée déterminée. Bien que n'étant pas directement lié à la réforme du code du travail, ce conflit permet de mieux en comprendre les enjeux.

La grève de septembre 1994 à Kafr al-DawwârLe contexte

5 Avec 12.000 employés, la « Filature fine » de Kafr al-Dawwâr est la seconde entreprise textile d'Égypte après sa consœur de Mahalla al-Kubra. Fondée comme cette dernière par le groupe bancaire nationaliste "Misr » (Égypte) dans les années 30, en période de chute des cours mondiaux du coton égyptien et de fermeture du marché local aux importations de tissu, elle a été conçue sur le modèle paternaliste des grandes usines européennes de la fin du XIXe siècle. Complexe intégré de production à grande échelle fondé sur une division taylorienne du travail, l'entreprise comprend dans son enceinte la cité ouvrière, l'hôpital, les écoles, les commerces et centres de loisirs destinés à permettre aux ouvriers de "se consacrer pleinement à leur tâche productive". Spécialisée dans la fabrication du fil et du tissu de coton écru, la «Filature fine » est représentative des problèmes de l'industrie cotonnière égyptienne, à savoir la transformation en grande quantité d'une matière première de luxe en produits finis bon marché. Située en plein milieu des champs de coton, elle ne peut utiliser des cotons importés moins chers. Dépourvue d'équipements de teinture et d'impression, elle doit sous-traiter la finition de ses produits à une entreprise «sœur » et voisine, «Misr alBayda pour la teinture et la finition», dont l'essentiel de l'activité dépend des commandes de la «Filature fine ».

6 Comme les autres usines textiles publiques, elle a dû fournir jusqu'en 1974 les produits alimentant les échanges de troc avec les pays du COMECON et approvisionner le marché local en produits textiles bon marché, sans se voir allouer par le plan les moyens de renouveler son parc de machines. Elle a bénéficié en 1976, avec sa consœur de Mahalla al-Kubra, d'un des deux premiers prêts extérieurs destinés à l'industrie égyptienne : 
Misr Mahalla a reçu celui de l'Aide américaine et Misr Kafr al-Dawwâr celui de la Banque mondiale et de l'Arab Fund for Economic and Social Development (AFESD). Le premier paradoxe de ces deux prêts fut d'imposer la construction de bâtiments supplémentaires pour accueillir les nouvelles machines et, par là-même, d'entraîner une extension des capacités de filature des deux mastodontes textiles égyptiens. Le second paradoxe fut de permettre l'extension des capacités de filature au milieu des principales zones de culture du coton, favorisant l'augmentation de sa transformation locale aux dépens de son exportation. Le prêt fut accordé par la Banque mondiale pour un taux annuel privilégié de $4 \%$ à l'État égyptien, qui le sous-traita au taux moins privilégié de $9 \%$ en parts égales entre la Filature et la Teinturerie, selon un contrat stipulant que le montant du service de la dette en livres égyptiennes (LE) serait calculé au taux de change en vigueur au moment du remboursement.

7 Le retrait du prêt de l'AFESO consécutif à la rupture des relations entre l'Égypte et ses créanciers arabes après Camp David obligea la Filature fine et al-Bayda' à emprunter les sommes manquantes à l'achèvement du projet-joint. Un incendie allait retarder la mise en activité de la nouvelle unité de filature de Kafr al-Dawwâr, alors que les intérêts couraient toujours. Les remboursements arrivèrent à échéance alors que l'entreprise se lançait dans un vaste programme de remplacement de ses métiers à tisser et que le prix de sa principale matière première, le coton, croissait rapidement. De surcroît, la firme, fidèle à la conception paternaliste du rôle régional de la grande entreprise, participa généreusement au financement des activités culturelles, syndicales et administratives locales, à la création d'entreprises textiles mixtes ainsi qu'à la rénovation et à l'extension des infrastructures sanitaires, d'habitation et sportives propres aux employés de l'entreprise. Cette générosité, associée à une politique très souple de gestion de la main-d'œuvre et des rapports avec la clientèle, favorisa probablement l'élection au Parlement du directeur de l'entreprise face à un syndicaliste local nassérien pourtant populaire. Elle assura la paix sociale dans une localité qui s'était rendue célèbre en septembre 1984 par la "grève de Ramadan ", première d'une série de conflits du travail qui ébranlèrent le secteur textile pendant trois années consécutives. En contrepartie, les dettes de l'entreprise s'élevaient, fin 1991, à 300 millions LE pour un chiffre d'affaires de 360 millions LE. Le découvert bancaire constituait $30 \%$ de cet endettement.

8 Avec la réorganisation du secteur public textile, cotonnier et de commerce de détail en trois sociétés holding officiellement concurrentes et solidaires de fait sous l'autorité du même Ministère du Secteur public des affaires, la Filature fine de Kafr al-Dawwâr changea de holding de tutelle et se vit attribuer un nouveau directeur. Surnommé affectueusement par le premier ministre, selon les dires du journal al-Sha'b, «le bulldozer du textile», le nouveau patron déclara prendre en charge l'assainissement financier de la firme, en commençant par supprimer tous les versements de fonds à des organismes extérieurs.

9 La destruction, pendant l'été 1993, de la récolte et des stocks de coton en Asie permit à la Filature fine comme à l'ensemble du secteur cotonnier égyptien d'écouler ses stocks et de transformer ses pertes financières en un excédent temporaire au cours de la saison 1993-94. Subjugués par le caractère de "juste despote » du nouveau maître de Kafr al-Dawwâr, contrastant avec la bonhomie dispendieuse de son prédécesseur, les membres de la commission syndicale élue de l'entreprise approuvèrent le système de primes et sanctions mis en place pour restaurer la discipline dans l'établissement et la 
réorganisation du travail destinée à réduire les gaspillages de temps et de matière première. Soutenu par sa base comme par ses supérieurs, le «sauveteur de Kafr alDawwâr ", comme le nommaient les syndicalistes locaux, poussa la logique de son plan de redressement jusqu'à priver 1.600 ouvriers de 3 mois de primes pour retards et congés maladies, à déplacer plusieurs chefs de département, à licencier 2,000 employés sous contrat temporaire mais renouvelé pour certains depuis six années consécutives, et à suspendre « à vie » le versement des primes à 300 ouvriers récalcitrants.

Le conflit et ses suites directes

10 Généralisées à l'ensemble des secteurs, ces mesures déclenchent, le vendredi 30 septembre 1994 en début d'après-midi, l'occupation de l'usine par l'équipe du matin sortante, à laquelle se joint l'équipe de l'après-midi. Le lendemain, la police encercle l'usine et coupe l'électricité. Le gouverneur, auquel se sont joints le ministre du Travail et le secrétaire général du syndicat textile, obtient la sortie des grévistes contre la promesse de la suppression des mesures de licenciement, de déplacement et de suppression de primes ainsi que la suspension du directeur. Mais les forces de sécurité, qui occupent les rues de la cité ouvrière avec des chars anti-émeute, perdent leur sang froid et tuent trois personnes extérieures à l'usine, dont un enfant sortant de l'école, touché par une balle perdue. Des habitants des environs bloquent alors la voie ferrée qui relie Kafr al-Dawwâr à Alexandrie en la recouvrant de pierres. 70 personnes sont arrêtées, dont 14 ouvriers, puis relâchées sous caution. A l'aube du dimanche 2 octobre, la Sécurité d'État arrête 24 ouvriers à leur domicile, sur listes fournies par la direction de l'entreprise.

11 L'après-midi du même jour, les ouvriers de l'équipe du matin de la société voisine d'alBayda' se rassemblent à la sortie de l'usine en proclamant leur soutien aux grévistes de la Filature fine emprisonnés. Ces derniers seront relâchés sous caution quelques heures plus tard. La Filature fine est mise en congé obligatoire et fermée pendant une semaine et la ville de Kafr al-Dawwâr tombe sous état de siège. La semaine suivante, 4.000 ouvriers (soit 1/5 du personnel, selon le journal al-Sha'b) de l'usine sœur de Mahalla alKubra se réunissent devant le local de la commission syndicale pour présenter à celle-ci leurs revendications. Ces dernières concernent, comme à Kafr al-Dawwâr, la modification du régime des primes et sanctions et l'application du système de distribution mensuelle de denrées alimentaires, pratiqué dans les autres branches industrielles, en remplacement de la prime de repas monétaire fixée à 12 LE par mois. A la suite de ce rassemblement, 11 syndicalistes et un enseignant de l'école technique textile, appartenant aux partis du Travail (nassérien-islamiste), du Rassemblement (nassérien-marxiste) et nassérien sont arrêtés et incarcérés à la prison de Tanta, pour éviter que se reproduisent les événements de Kafr al-Dawwâr.

12 Face aux risques d'extension du conflit, le gouvernement égyptien adopte une stratégie d'apaisement. Le directeur de Kafr al-Dawwâr, réintégré dans ses fonctions, réunit les chefs de département et met en place des commissions d'étude des problèmes de la main-d'œuvre. Son retour provoque néanmoins, selon le journal al-Sha'b, des arrêts de travail et manifestations au sein de l'entreprise et dans la ville de Kafr al-Dawwâr, les grévistes réclamant l'embauche définitive des ouvriers sous contrat temporaire et le maintien des primes. Les pertes de l'entreprise, aggravées par la grève et ses suites, atteindraient, selon le même journal, 1 milliard LE, et son découvert bancaire 750 millions LE. Le ministre du Secteur public, accompagné du ministre du Travail, entreprend dans la même semaine une série de réunions d'écoute et de clarification 
avec les secrétaires de commissions syndicales des entreprises publiques du gouvernerat d'al-Bahîra, de celles de la ville d'Alexandrie et du gouvernorat limitrophe d'al-Gharbiyya, ainsi que dans la banlieue industrielle du Caire à Shubra al-Khayma. Le mercredi 19, le ministre du Travail et le secrétaire général du syndicat textile réunissent au Caire les secrétaires de commissions syndicales de toutes les entreprises textiles. La présentation du nouveau code du travail au Parlement, prévue pour le dernier trimestre 1994 selon l'accord passé entre le gouvernement égyptien et le BIT, est reportée à une date ultérieure.

Relecture de la grève en Égypte dans le contexte de la libéralisation du marché du travail

13 La grève, interdite jusqu'à présent par la loi égyptienne mais dépénalisée depuis la ratification par l'Égypte des conventions internationales, est encore considérée par les pouvoirs publics comme le symptôme d'une erreur de gestion, tandis que les ouvriers ne la conçoivent que comme ultime recours à une situation d'injustice caractérisée. Institutionnalisée par les codes du travail successifs, la gestion paternaliste de la relation de travail dans le secteur public garantit au travailleur l'emploi et le minimum vital en échange d'une soumission totale à son employeur. En contrepartie de l'obligation morale de subsistance que s'impose l'État vis-à-vis de son employé, il s'autorise à exercer une répression féroce de tout écart au respect de l'ordre établi. Le contenu des tâches n'étant pas défini par le contrat de travail mais par la relation paternaliste liant les deux parties, c'est le travailleur, en fonction de ses diplômes et de son ancienneté, qui est rémunéré, et non son travail. La totale dichotomie qui en résulte entre le salaire et le travail devient un obstacle au renforcement de la discipline, aux gains de productivité et à la baisse des coûts rendus nécessaires par la récession intérieure.

En cela, la transformation de la relation de travail est un élément clé du processus de libéralisation en cours de l'économie égyptienne. Pour que le prix du travail s'établisse, comme celui des autres marchandises, en fonction de l'offre et de la demande, l'employeur doit être dégagé de toutes les obligations morales inclues dans le pacte instauré par le régime nassérien.

C'est la raison pour laquelle le projet de code du travail, répondant aux vœux des chefs d'entreprise, supprime toutes les entraves juridiques au licenciement. Voulant respecter l'équilibre libéral entre la liberté de l'employeur et celle de l'employé, il introduit dans la législation égyptienne la notion de négociation collective et le droit de grève. Mais fidèle à la tradition paternaliste et respectueux de l'équilibre des forces sur la scène politique égyptienne, il pose de strictes limitations à l'exercice de ce droit et restreint la représentation collective des ouvriers à la direction du syndical unique de branche. Le projet de loi sur les syndicats, complémentaire du projet de code du travail, prévoit en outre l'allongement des mandats des délégués des instances supérieures. La négociation collective prévue par le législateur égyptien, se substituant à la gestion administrative directe de l'État, fait ainsi intervenir des cadres syndicaux professionnalisés et peu sensibles aux pressions de leur base. Il prévoit également l'intermédiation d'un "médiateur", sur le modèle français, en cas d'échec des procédures tripartites. Durant la négociation et l'intervention du médiateur, ainsi qu'au cours de la période de validité de la convention collective précédente, la grève est interdite par le nouveau code du travail. Ayant réduit à l'extrême le droit de grève et la représentation ouvrière en cas de différend, le législateur vise à renforcer la position de 
l'employeur et de l'État dans les conflits et à limiter les «acquis » inscrits dans les conventions collectives en période d'austérité et de restructuration. La "négociation collective» promue par la nouvelle loi vise de fait à décentraliser la gestion paternaliste des relations de travail, par substitution des chefs d'entreprise, des syndicats et des délégations régionales du Ministère du Travail à l'État central.

La grève, régulateur des salaires sur le marché du travail

Le nouveau code du travail, par les procédures longues et complexes de négociation collective et les restrictions portées au droit de grève, cherche à limiter l'occurrence des arrêts de travail collectifs dans une période où les conflits sociaux risquent de se multiplier. En effet, la grève, bien qu'interdite, a été largement pratiquée depuis 1968, particulièrement au cours des deux phases d'austérité qui ont précédé puis suivi le boom pétrolier (1979-85). Pendant cette courte période de vaches grasses, l'émigration et l'essor de l'activité intérieure offrent des solutions individuelles à la dégradation des salaires du secteur public et l'activité revendicative décline relativement au cours de la décennie 1968-78. Au contraire, au cours de cette dernière et de la phase de récession qui suit la baisse des prix du pétrole (1985-94), les mouvements de grève se multiplient, permettant de relever les salaires réels lorsque les mécanismes légaux de négociation collective n'avaient pas suffi à empêcher leur dégradation. Dans le secteur public textile, la corrélation entre les conflits sociaux ouverts et les variations de salaires sont très nettes. Le salaire réel moyen textile, après avoir stagné jusqu'en 1981, croît de près de $20 \%$ au cours de la seule année 1982 pour décliner à nouveau en 1983 et 1985 et retomber en 1990 au-dessous de son niveau de 1975. Les effectifs suivent une progression plus régulière jusqu'en 1982, puis décroissent rapidement pour tomber dès 1986 au-dessous du chiffre de 1975. L'emploi textile de 1994 ne représente plus que $60 \%$ de celui de 1982 et la masse salariale a été divisée par deux en termes réels.

La première grande vague de grèves de la période post-1967 débute à Mahalla al-Kubrâ (1971), se poursuit à Hilwân (1974) puis reprend à Mahalla al-Kubrâ (1975). Elle culmine en 1976 avec les premières élections syndicales organisées depuis 1964. Elles sont l'occasion d'une vaste campagne politique de l'opposition de gauche, et de nombreux syndicalistes communistes ou nassériens sont élus. Les émeutes de janvier 1977 provoquées par la hausse du prix du pain débutent par une manifestation d'ouvriers de la filature de Hilwân. Le gouvernement opte alors pour la politique de la carotte et du bâton : d'une part, la prison et l'ouverture d'une série de procès pour les leaders syndicaux et les militants politiques et, d'autre part, la hausse des salaires et la possibilité offerte aux ouvriers des entreprises les plus contestataires d'être reclassés avec les mêmes salaires dans des fonctions administratives sur le lieu de leur choix. Cette stratégie s'avère payante, puisque les effectifs ouvriers de l'usine de Hilwân diminuent de 10 \% entre 1976 et 1980 et que le salaire moyen baisse légèrement en 1979.

18 La deuxième élection syndicale en 1979 réactive le mouvement revendicatif, malgré le maintien des lois d'exception et les annulations des candidatures d'opposition par le procureur général. Le rejet par la gauche des accords de Camp David et de la normalisation des relations avec Israël exacerbe les tensions politiques. Les arrestations de militants politiques et syndicaux en 1981 sont davantage liées au débat politique qu'aux conflits de travail. Ces derniers disparaissent jusqu'en 1985, le développement de l'émigration et le boom économique à l'intérieur du pays ouvrent la porte à la recherche de solutions individuelles. Par ailleurs, la politique 
d'investissements massifs dans le secteur public industriel s'accompagne d'embauchés nettes et d'une hausse du salaire moyen réel entre 1975 et 1982. Enfin, l'assassinat du président Sadate est suivi d'une politique d'apaisement et de l'ouverture d'un « débat national » avec les différentes forces de l'opposition. Toutes les personnes incarcérées sont relâchées et les salaires réels connaissent une certaine progression. Le secteur public textile, où le salaire moyen est en croissance continue jusqu'en 1982 (excepté l'année 1979), ne connaît pas de grève notoire avant 1985. Les années 1983 et 1984 correspondent au début de la réorganisation du secteur public textile sous l'autorité de l'Organisation des textiles, dont la direction est confiée à un homme "à poigne » qui décide de bloquer les salaires nominaux, ce qui se traduit par deux années de baisse du salaire réel en 1983 et 1984.

19 La tenue des troisièmes élections syndicales en 1983 voit renaître le mouvement revendicatif exacerbé par les réductions de salaires. En 1985 éclate à al-Mahalla alKubrâ et à l'usine ESCO du Caire un conflit qui durera plusieurs semaines, portant sur le paiement du congé du vendredi, prévu par la loi mais non appliqué dans la branche textile. Le jugement, favorable aux ouvriers, fera jurisprudence et sera généralisé aux 31 firmes publiques. Il se traduit la même année par une hausse du salaire moyen qui sera la dernière de la période post-1967. Dans les autres branches, l'activité revendicative et syndicale reprend dès 1986, touchant d'abord les transports puis s'étendant aux industries mécaniques et électriques, à la faveur des élections syndicales de 1987. Cette nouvelle vague s'achève par la grève avec occupation de l'usine sidérurgique de Hilwân en 1989. Les revendications portaient essentiellement sur les primes de rendement et sur la transformation de la prime de repas monétaire en un « panier » de provisions mensuel. La brutalité de la répression, justifiée par le caractère massif de la mobilisation, fait de ce conflit un symbole. Elle marque les limites de l'affrontement tolérable par le gouvernement, qui se trouve alors en pleine crise de paiements et entame des négociations avec le FMI. De 1986 à 1991, le salaire réel diminue chaque année jusqu'à atteindre en 1991 un niveau inférieur à celui de 1975. Sa baisse se poursuit en 1992,1993 et 1994. Pour obtenir ce résultat, les dirigeants des firmes textiles, encouragés par la direction de leur organisation sectorielle, ont limité les nouveaux recrutements à des contrats à durée déterminée, renouvelables chaque année. Le personnel, d'autre part, s'est féminisé, tant à cause du bas niveau des rémunérations que par substitution d'activités «de précision" aux activités "de force ». Le développement du prêt-à-porter et la réduction du tissage ainsi que la modernisation des machines permettent d'utiliser davantage de femmes, aux salaires inférieurs à ceux des hommes. La part du salaire au rendement étant plus importante dans le textile que dans les autres branches d'activité, la diminution de l'activité s'est traduite rapidement par une réduction des coûts salariaux. En septembre 1990, moins d'un an après l'occupation spectaculaire de l'usine sidérurgique de Hilwân, une grève éclate de nouveau dans plusieurs usines à Mahalla al-Kubrâ, après la suppression de la prime de rentrée scolaire. Elle se solde par l'arrestation et le déplacement en province d'une dizaine de délégués et par le rétablissement de la prime. Contrairement aux conflits antérieurs à 1985, ce dernier ne se solde pas par une hausse du salaire réel. Il est trop tôt pour pouvoir connaître l'impact de la grève de septembre 1994 sur l'évolution des salaires de la Filature fine de Kafr al-Dawwâr, mais il semble probable que cette dernière puisse tout au plus freiner une réduction apparemment inéluctable. Il est clair que les conflits du travail postérieurs à 1985, dans le textile comme dans les autres branches, ont changé de fonction. Il ne s'agit plus pour les salariés de maintenir 
leur part d'un revenu national en constante croissance, mais de limiter la réduction de leur part d'un revenu national lui-même en diminution.

L'enjeu du nouveau code unifié du travail : abaisser le coût du travail

21 Au niveau de la seule branche textile, les salaires, qui consommaient le quart des recettes des ventes des firmes publiques en 1975, voient leur part s'élever au cours des 10 années suivantes jusqu'à atteindre $40 \%$ de ces recettes. Pendant le même temps, la part des produits intermédiaires croît de $55 \%$ à $70 \%$, celle des paiements d'intérêts s'élève de $1 \%$ à $9 \%$, celle des provisions pour amortissement double de $4 \%$ à $8 \%$, au détriment de celle des taxes, impôts et bénéfices distribuables qui tombe de $18 \%$ à $9 \%$. Le déficit courant atteint $20 \%$ des recettes en 1985. Malgré une politique d'investissement ambitieuse et l'augmentation des prix des intrants locaux, le déficit est ramené à $5 \%$ des recettes de ventes en 1991. Grâce à une réduction draconienne de la part des salaires à $20 \%$ du total, tandis que les autres postes conservent leur poids relatif du milieu des années 80 . Après l'application de la loi 203 et le relèvement des taux d'intérêt intérieurs, la part du déficit relativement au chiffre d'affaires s'accroît à nouveau, celle des salaires restant stable. Une nouvelle ponction sur les salaires, qui ne consomment plus que $20 \%$ des recettes, n'aura d'impact réel sur la situation financière de l'entreprise qu'à condition d'opérer des réductions draconiennes d'effectifs allant du quart à la moitié de ces derniers, ou d'abaisser fortement les salaires nominaux.

C'est ce que prévoit le programme d'ajustement structurel, à travers la restructuration du secteur public et grâce au nouveau code du travail. Ce dernier devrait permettre à la fois de licencier et d'abaisser les salaires sans restriction juridique notoire. Il n'en reste pas moins - et la grève de Kafr al-Dawwâr est là pour le rappeler-que l'application des mesures d'ajustement autorisées par la législation actuelle ou future ne se fera pas sans conflits sociaux majeurs. Vu l'importance relative du coût des matières premières, on peut s'interroger sur la possibilité d'effectuer des choix de produits plus économes en matières premières et de la mise en œuvre de productions « juste à temps » réduisant le volume des matériaux (70 \% des recettes en 1990 et 1991), des déchets de production (2 à $3 \%$ des recettes), des stocks et des ventes à crédit (respectivement $55 \%$ et $20 \%$ du chiffre d'affaires), sans compter la part des dettes intérieures et extérieures et des intérêts qui en découlent (respectivement $130 \%$ et $7 \%$ du total des recettes) dont une grande part revient aux achats locaux et extérieurs de matières premières. Mais la modification radicale des systèmes de production et de commercialisation est plus difficile à mettre en œuvre, avec des cadres formés depuis trente ans à la réalisation des objectifs quantitatifs dans un marché protégé, que les réductions de salaires et les dégraissages d'effectifs. Il est probable que les conflits sociaux violents que ces politiques entraîneront dans les grandes entreprises inciteront ces dernières à rationaliser leur mode de fonctionnement, comme ce fut le cas dans l'automobile en France dans les années 80 . En taillant une large brèche dans la protection de «l'emploi et du salaire acquis » héritée de la période nassérienne, le nouveau code du travail crée les conditions de la réactivation du mouvement ouvrier et de l'adaptation de l'appareil productif à la concurrence internationale. 
INDEX

Mots-clés : travail, économie, libéralisation, grève, textile

\section{AUTEUR}

FRANÇOISE CLÉMENT

CEDEJ 